4. Van Duin D, Doi Y. The global epidemiology of carbapenemase-producing Enterobacteriaceae. Virulence. 2017; 8(4):460469.

5. Ocampo AM, Chen L, Cienfuegos AV, Roncancio G, Chavda KD, Kreiswirth BN, Jiménez JN. A Two-Year Surveillance in Five Colombian Tertiary Care Hospitals Reveals High Frequency of Non-CG258 Clones of Carbapenem-Resistant Klebsiella pneumoniae with Distinct Clinical Characteristics. Antimicrob Agents Chemother. 2015; 60(1):332-42.

6. Pereira PS, Borghi M, de Araújo CF, Aires CA, Oliveira JC, Asensi MD, Carvalho-Assef AP. Clonal Dissemination of OXA-370-Producing Klebsiella pneumoniae in Rio de Janeiro, Brazil. Antimicrob Agents Chemother. 2015; 59(8):4453-6.

Correspondencia: Jose A. Gonzales-Zamora

Dirección: 1120 NW 14th Street, Suite 863B. Miami, Fl 33136. EE.UU.

Teléfono: (001) 706-284-3510

Correo electrónico:jxg1416@med.miami.edu

\section{SOBRESTIMACIÓN DE ALERGIA ALIMENTARIA REPORTADA POR PADRES EN UN CENTRO PERUANO DE ALERGIA, ASMA E INMUNOLOGÍA}

\section{OVERESTIMATION OF FOOD ALLERGIES REPORTED BY PARENTS IN A PERUVIAN ALLERGY, ASTHMA, AND IMMUNOLOGY CENTER}

\section{Daniel Mendoza-Quispe ${ }^{1,2, a}$, Liliana Alvarez ${ }^{1,2, a}$, César A. Galván ${ }^{3,4, b}$}

Sr. Editor. La alergia alimentaria $(\mathrm{AA})$ es una reacción adversa perjudicial de origen inmunológico. Afecta al 2-4 \% de la población mundial, al 6-8\% de niños menores de un año, ysu prevalencia sigue incrementándose significativamente desde las últimas décadas ${ }^{(1)}$. La búsqueda del término «alergia» en la base Scielo Perú (www.scielo.org.pe) muestra doce resultados, y sólo uno se relaciona con AA. La prevalencia y los alimentos causales varían entre países, el manejo está

Facultad de Medicina, Universidad Nacional Mayor de San Marcos. Lima, Perú. Asociación para el desarrollo de la investigación en Ciencias de la Salud. Lima, Perú.

3 Centro de Referencia Nacional de Alergia, Asma e Inmunología. Instituto Nacional de Salud del Niño. Lima, Perú.

4 Sociedad Peruana de Inmunología. Lima, Perú.

a Médico cirujano; ${ }^{b}$ médico especialista en Alergología e Inmunología Clínica Recibido:26/06/2018 Aprobado:28/11/2018 En línea: 21/12/2018

Citar como: Mendoza-Quispe D, Alvarez L, Galván CA. Sobrestimación de alergia alimentaria reportada por padres en un centro peruano de alergia, asma e inmunología. Rev Peru Med Exp Salud Publica. 2018;35(4):708-10. doi: 10.17843/rpmesp.2018.354.3763. centrado en la restricción dietética del alimento causal (2). Un diagnóstico equivocado de AA, del que frecuentemente participan los padres, conduciría a una innecesaria restricción dietética, que podría afectar el desarrollo nutricional y psicológico del niño ${ }^{(2,3)}$. Por ello, el objetivo del estudio fue evaluar la estimación de AA reportada por padres asistentes a un centro de alergia, asma e inmunología.

Desde setiembre de 2016 a enero de 2018 aplicamos una encuesta semiestructurada a padres de 328 pacientes menores de 18 años, que acudieron por primera vez a consulta ambulatoria al Centro de Alergia, Asma e Inmunología del Instituto Nacional de Salud del Niño ubicado en Lima. Los pacientes fueron evaluados por un médico alergólogo e inmunólogo clínico (anamnesis, examen físico y pruebas de alergia, en caso de ser necesarias) para determinar su diagnóstico definitivo. Se obtuvo el consentimiento informado verbal de los padres. Los datos fueron analizados de forma descriptiva usando el programa estadístico SPSS versión $24 \circledast$.

De 328 pacientes evaluados, el 59,8 \% fueron varones, el $27,1 \%$ tenía entre 0-2 años, el 28,4 \% tenía entre 3-5 años, y el $44,5 \%$ tenía entre 6-18 años. Asimismo, el 40,9 \% (134/328) de padres refirió que su hijo era alérgico a algún alimento (cítricos, leche y/o derivados, y alimentos con preservantes, entre los más frecuentes). Luego de ser evaluados sólo se confirmaron tres casos $(0,9 \%, 3 / 328)$, todos alérgicos a la proteína de leche de vaca. La manifestación que los padres más frecuentemente asociaron a AA fueron ronchas, tos y prurigo. El 57,5 \% (77/134) refirió que el diagnóstico le había sido dado por un médico. El 48,5 \% (159/328) refirió que alguna vez un médico le recomendó restringir algún alimento por sospecha de AA (Tabla 1).

Nuestro hallazgo es mayor a lo reportado por Ontiveros et al. quienes encuestaron a 1049 padres de niños mexicanos, además el 5,5\% respondió que su hijo tuvo alguna vez $A A$, y el 4,9\% refirió que un médico alguna vez les dijo que su hijo tenía $A A^{(2)}$. Un metaanálisis europeo encontró una prevalencia de 5,9\% de AA cuando esta fue medida por autoreporte de los padres y 0,9\% cuando la AA era diagnosticada por test de provocación oral ${ }^{(1)(3)}$.

La mayoría de reacciones alérgicas son debidas a pocos alimentos como el maní, nueces, huevo, leche, pescado y mariscos ${ }^{(4)}$. Sin embargo las creencias pueden variar entre países, padres mexicanos reportaron que la leche, camarones y otros mariscos pueden causar alergia; padres noruegos reportan que las frutas, chocolate, nueces ${ }^{(4)}$ pueden causar alergia. En nuestra muestra encontramos la creencia de que los cítricos pueden causar mayoritariamente alergias; aunque se ha identificado sensibilización a cítricos, generalmente por reacción cruzada con proteínas homólogas a las del polen, la alergia es raramente observada ${ }^{(5)}$. 
Tabla 1. Frecuencia de alergia alimentaria y sus características, reportado por padres de niños que buscan atención en el Centro de Referencia Nacional de Alergias, Asma e Inmunología del Instituto Nacional de Salud del Niño (2016-2018)

\begin{tabular}{|c|c|c|}
\hline Pregunta & Frecuencia & Porcentaje \\
\hline $\begin{array}{l}\text { 1) ¿Cree usted que su hijo es } \\
\text { alérgico a algún alimento? * }\end{array}$ & $134 / 328$ & 40,9 \\
\hline Sí, a los cítricos & $43 / 134$ & 32,1 \\
\hline Sí, a la leche y/o derivados & $33 / 134$ & 24,6 \\
\hline $\begin{array}{l}\text { Sí, a los alimentos con } \\
\text { preservantes }\end{array}$ & $23 / 134$ & 17,2 \\
\hline Sí, al huevo & $18 / 134$ & 13,4 \\
\hline Sí, al chocolate & $17 / 134$ & 12,7 \\
\hline Sí, a las golosinas & $15 / 134$ & 11,2 \\
\hline Sí, al pescado & $13 / 134$ & 9,7 \\
\hline Sí a las frutas (no cítricos) & $12 / 134$ & 9,0 \\
\hline Sí, a los mariscos & $5 / 134$ & 9,0 \\
\hline Sí, otros & $28 / 134$ & 20,9 \\
\hline \multicolumn{3}{|l|}{$\begin{array}{l}\text { 2) ¿Qué síntomas cree usted que } \\
\text { presenta/presentó su hijo? * }\end{array}$} \\
\hline Ronchas & $65 / 134$ & 48,5 \\
\hline Tos & $25 / 134$ & 18,7 \\
\hline Prurigo & $22 / 134$ & 16,4 \\
\hline Escozor & $18 / 134$ & 13,4 \\
\hline Rinorrea & $14 / 134$ & 10,4 \\
\hline Otros & $22 / 134$ & 16,4 \\
\hline \multicolumn{3}{|l|}{$\begin{array}{l}\text { 3) ¿Quién le informó que esos } \\
\text { síntomas corresponden a AA? * }\end{array}$} \\
\hline Un médico & $77 / 134$ & 57,5 \\
\hline Un familiar & $25 / 134$ & 18,7 \\
\hline Otros medios & $39 / 134$ & 29,1 \\
\hline $\begin{array}{l}\text { 4) ¿Alguna vez un médico le } \\
\text { restringió a su hijo algún alimento } \\
\text { por sospecha de AA? }\end{array}$ & $159 / 328$ & 48,5 \\
\hline Sí, los cítricos & $78 / 328$ & 49,1 \\
\hline $\begin{array}{l}\text { Sí, los alimentos con } \\
\text { preservantes }\end{array}$ & $55 / 328$ & 34,6 \\
\hline Sí, leche y/o derivados & $51 / 328$ & 32,1 \\
\hline Sí, el chocolate & $23 / 328$ & 14,5 \\
\hline Sí, el pescado & $22 / 328$ & 13,8 \\
\hline Sí, el huevo & $20 / 328$ & 12,6 \\
\hline Si, otros & $51 / 328$ & 15,5 \\
\hline $\begin{array}{l}\text { 5) Casos confirmados de AA en } \\
{\text { el estudio }{ }^{+}}\end{array}$ & $3 / 328$ & 0,9 \\
\hline
\end{tabular}

AA: Alergia alimentaria

* En estas preguntas, el encuestado pudo haber respondido más de una alternativa.

† Luego de entrevista clínica, prueba de alergia cutánea (prick test) positivo, y slgE (IgE específico) positivo, realizado por médico alergólogo e inmunólogo clínico
Los síntomas de AA son predominantemente de la piel, seguidos por el tracto gastrointestinal y respiratorio. El prurigo, frecuentemente mencionado en nuestro estudio, no es una presentación de $\mathrm{AA}^{(4)}$.

Dirceu et al. encontraron errores en el diagnóstico y manejo de AA en 895 pediatras brasileños, como asociar el consumo de chocolate, la presencia de sibilancias aisladas o la otitis media con AA, y el uso de leche de cabra o de soya como sustitutos de la leche de vaca. Además, el $74,8 \%$ asoció los aditivos químicos con $\mathrm{AA}{ }^{\left({ }^{(6)}\right.}$. Estos saborizantes (ej. tartrazina) y preservantes (ej. glutamatos) pueden causar reacciones adversas del tipo intolerancia y no alérgicas, por lo que no existen mecanismos inmunológicos involucrados ${ }^{(4)}$.

De esta forma, los médicos también contribuyen al sobrediagnóstico de AA, probablemente por proteger al niño de una potencial reacción alérgica, sin considerar que un tercio de niños sufren de bullying asociado a su diagnóstico, disminución de su calidad de vida, y que la reducción de ingesta de nutrientes interferirá en su crecimiento físico ${ }^{(3)}$.

La principal limitación observada en el presente estudio es el sesgo de selección, el cual podría haber afectado la magnitud de ocurrencia del fenómeno observado (percepción de AA). Es razonable pensar que los padres que llevan a sus hijos a un centro de alergia tengan mayores sospechas de AA, lo que finalmente podría haber condicionado la sobreestimación descrita.

Nuestro estudio sugiere que los padres que acuden a un centro de alergia sobreestiman la presencia de una AA en sus hijos, lo que podría ocasionar restricciones dietéticas erróneas que afectan el correcto desarrollo nutricional y psicológico del niño. Sugerimos aumentar las comunicaciones científicas sobre AA y establecer guías de diagnóstico y manejo adecuado sobre esta condición.

Contribuciones de autoría: DMQ, LA y CAG han participado en la concepción y diseño del artículo, recolección, análisis e interpretación de datos y redacción del artículo. Todos aprobaron la versión final.

\section{Fuentes de financiamiento: Autofinanciado}

Conflictos de interés: Ninguno

\section{REFERENCIAS BIBLIOGRÁFICAS}

1. Nwaru B, Hickstein L, Panesar SS, Muraro A, Werfel T, Cardona V, et al. The epidemiology of food allergy in Europe: a systematic review and meta-analysis. Allergy. 2014;69(1):6275. doi: 10.1111/all.12305.

2. Ontiveros N, Valdez-Meza EE, Vergara-Jiménez MJ, Canizalez-Román A, Borzutzky A, Cabrera-Chávez F. Parent-reported prevalence of food allergy in Mexican schoolchildren: 
A population-based study. Allergol Immunopathol (Madr). 2016;44(6):563-570. doi: 10.1016/j.aller.2016.03.003.

3. Coon ER, Quinonez RA, Moyer VA, Schroeder AR. Overdiagnosis: How Our Compulsion for Diagnosis May Be Harming Children. Pediatrics. 2014;134(5):1013-23. doi: 10.1542/peds.2014-1778.

4. Burks AW, Tang M, Sicherer S, Muraro A, Eigenmann PA, Ebisawa M, et al. ICON: Food allergy. J Allergy Clin Immunol. 2012;129(4):906-20. doi: 10.1016/j.jaci.2012.02.001.

5. Iorio RA, Del Duca S, Calamelli E, Pula C, Lodolini M, Scamardella F, et al. Citrus Allergy from Pollen to Clinical Symptoms. PLoS One. 2013;8(1):e53680. doi: 10.1371/ journal.pone.0053680.

6. Sole D, Jacob CMA, Pastorino AC, Porto Neto A, Burns DA, Sarinho ESC, et al. O conhecimento de pediatras sobre alergia alimentar: estudo piloto. Rev Paul Pediatr. 2007; 25:311-6.

Correspondencia: Daniel Enrique Mendoza Quispe

Dirección: Parque Unión Panamericana 136. Lima, Perú

Teléfono: +51987829720

Correo electrónico: daniel91emq@gmail.com

\section{PERCEPCIÓN SOBRE PROMOCIÓN FARMACÉUTICA Y PRESCRIPCIÓN DE MEDICAMENTOS EN MÉDICOS DE LIMA, PERÚ 2016}

\author{
PERCEPTIONS ON PHARMACEUTICAL \\ PROMOTION AND MEDICINE PRESCRIPTION \\ OF PHYSICIANS IN LIMA, PERU 2016
}

\author{
Rafael O. Florián-Castro. 1,a.
}

Sr. Editor. En los últimos 50 años, los visitadores médicos son el principal canal de comunicación para transmitir información sobre mercadotecnia de productos farmacéuticos mediante la visita a médicos. Esta actividad representa el $60 \%$ de los gastos de ventas de la industria farmacéutica. En Estados Unidos la información brindada en la mercadotecnia de los visitadores médicos está regulada y supervisada para asegurar su consistencia con lo estipulado por la Agencia de Administración de

\footnotetext{
Escuela de Posgrado. Universidad San Ignacio de Loyola. Lima, Perú. Ingeniero, Magister y Doctor en Administración.

Recibido: 07/06/2018 Aprobado: 05/09/2018 En línea: 21/12/2018
}

Los resultados son parte de la tesis doctoral de Florián Castro R. (2017). Relación entre promoción farmacéutica y prescripción de medicamentos en Perú [Tesis doctoral]. Lima: Universidad San Ignacio de Loyola. Escuela de Postgrado 2017. Citar como: Florián-Castro RO. Percepción sobre promoción farmacéutica y prescripción de medicamentos en médicos de Lima, Perú 2016. Rev Peru Med Exp Salud Publica. 2018;35(4):710-1.doi:10.17843/rpmesp.2018.354.3718.
Alimentos y Medicamentos (FDA). En el 2010, el $11 \%$ de los médicos estadounidenses tenían restringido el contacto con visitadores médicos y $34 \%$ tenían algunas restricciones para contactar con los visitadores médicos ${ }^{(1)}$.

Los medicamentos son promocionados por los visitadores médicos mediante la exposición de sus principales características y bondades, o la entrega de muestras médicas para persuadir a médicos de prescribirlos ${ }^{(2)}$; dichas actividades requieren que los visitadores médicos tengan formación y adiestramiento adecuados, además de integridad ética para presentar información sobre medicamentos y realizar otras actividades de promoción de sus productos de manera correcta y responsable ${ }^{(3)}$.

Los criterios éticos para la promoción de medicamentos constituyen un estándar para apoyar y fomentar una mejor atención de la salud mediante el uso racional de medicamentos. La promoción a cargo de los visitadores médicosdebeconsiderarunusoadecuadodemedicamentos, mediante una presentación objetiva de sus propiedades, sin exagerar sus características. En Yemen un estudio demostró que las relaciones entre médicos y la industria farmacéutica generan conflictos con los criterios éticos de promoción de medicamentos. Los médicos contribuyen a establecer actividades promocionales indeseables cuando solicitan diversos beneficios, a los que consideran tienen derecho, a cambio de prescribir medicamentos. Por otro lado, los bajos ingresos de los médicos contribuyen a que la industria farmacéutica ofrezca promociones y beneficios, como ofertas de viajes, alojamiento en hoteles y expectativas de algunas donaciones de fondos ${ }^{(4)}$. Es importante destacar que la situación en el Perú no es muy diferente, por las bajas escalas remunerativas.

Como parte de la tesis doctoral del autor, se realizó un estudio transversal mediante la utilización de un cuestionario de 24 preguntas para determinar la relación entre la promoción farmacéutica y la prescripción de medicamentos. El cuestionario incluyo un párrafo introductorio que indicaba al participante el carácter voluntario, la confidencialidad en el manejo de los datos recopilados y su consentimiento en usar estos para el estudio. El diseño del cuestionario se basó en la revisión de literatura científica, la validación de cinco expertos (midiéndose suficiencia, claridad, coherencia y relevancia con la $\mathrm{V}$ de Aiken) y aplicándose una prueba piloto a 24 médicos.

Queremos describir la respuesta de los médicos respecto a dos preguntas ¿Existe conflicto de tipoético entre la promoción farmacéutica y los médicos al prescribir medicamentos? y ¿La promoción farmacéutica (visita médica, muestras médicas, etc.) está debidamente regulada en el Perú por el MINSA y DIGEMID? La encuesta se realizó entre enero y abril del 2016 a 2731 médicos de alto nivel prescriptor (buena cantidad de recetas captadas por la auditoria CloseUp International) y que laboran en Lima Metropolitana. Los médicos participantes laboraban en centros de salud del MINSA, Essalud, clínicas 